\title{
Specific attentional effects reflected in the cardiac orienting response
}

\author{
JAMES W. BROWN \\ San Diego State University, San Diego, California 92115 \\ and
}

PHILIP A. MORSE, LEWIS A. LEAVITT, and FRANCES K. GRAHAM

University Hospitals, University of Wisconsin, Madison, Wisconsin 53706

\begin{abstract}
Twenty-four undergraduates listened to 60 -sec strings of synthetic speech syllables containing a syllable change in the middle of the string. No response was required but half of the subjects were instructed to "listen for a change." Instructions did not affect cardiac orienting to onsets or offsets of the strings but did determine whether or not orienting occurred to a change that all subjects reported hearing. The findings imply that a failure of nonverbal subjects to orient to stimulus change cannot be accepted as evidence that the change is not discriminated.
\end{abstract}

The use of an unconditioned orienting response as an indication of discrimination is an attractive possibility whenever verbal instructions or responses are inappropriate. According to Sokolov (1963), at least some components of an orienting response should accompany the detection of stimuli which are novel or unexpected. Since the cardiac component of orienting, heart rate (HR) deceleration (Graham \& Clifton, 1966), is relatively easy to record, a number of investigators have successfully demonstrated discrimination in infants by using cardiac orienting paradigms (e.g., Adkinson \& Berg, 1974; Berg, 1972; Moffitt, 1971). Commonly, such studies have presented one stimulus for several successive trials, sufficient to produce response decrement, and have then introduced the stimulus to be discriminated. Recovery of orienting on the changestimulus trial is considered evidence for discrimination. Leavitt, Morse, Brown, and Graham (1973) and Leavitt, Brown, Morse, and Graham (Note 1) have also used a "no-delay" modification of the paradigm which eliminates the memory load imposed by an intertrial interval. Since HR in response to a long-lasting stimulus usually returns to a steady baseline while the stimulus is still present, it is possible, by introducing a second stimulus immediately following a sufficiently longlasting first stimulus, to evoke a second orienting response to the change in stimulation.

The interpretation of positive results in these paradigms is clear; the occurrence of orienting to change stimuli implies that, at some level, subjects detected

This work was supported by the Grant Foundation, by Public Health Service Grants HD01490 and HD08240 and by Research Scientist Award K3-MH21762. Computing services were provided through Grant FR00249 to the Laboratory Computing Facility of the Medical School, University of Wisconsin. Address requests for reprints to Frances $\mathrm{K}$. Graham, University Hospitals, Madison. Wisconsin 53706. the change. What is not clear is the meaning of experiments which obtain negative results. Does the absence of an orienting response to stimulus change imply no discrimination (e.g., Maltzman \& Raskin, 1965; Vinogradova, 1965) or does it mean, instead, that a detected stimulus did not engage attention (Graham \& Clifton, 1966; Kagan \& Lewis, 1965)? Sokolov's description of orienting as a reflexive response to mismatch between current stimulus input and a neuronal model of past input could be interpreted to mean that orienting is an "automatic, mechanically produced accompaniment to any perceptible stimulus novelty" (Bernstein, 1969, p. 339). However, Sokolov (1969, p. 673) emphasized that the neuronal model contains "only those relationships of interest to the organism in adapting to its surroundings." This statement would appear to agree with Bernstein's position $(1969,1975)$ that orienting is elicited only by stimuli having "significance." A stimulus does, of course, have significance when it signals that a response is to be made, and orienting under such signal conditions is usually found to be more pronounced than under nonsignal conditions (e.g., Bernstein, Taylor, \& Weinstein, 1975). The question of interest here is whether, in a nonsignal situation requiring no overt response, every perceptible novel change elicits orienting. The sparse data available suggest that it does not, since Bernstein (1969) found that many subjects who correctly reported a change in visual-stimulus intensity following a 10-trial habituation series did not give orienting responses, indexed by an electrodermal response, on the change trial.

The study reported in the present paper employed the cardiac response to measure orienting and provides further support for the position that perception of change is not necessarily accompanied by orienting. The subjects were undergraduates who were presented 
with a synthetic speech change that failed to elicit orienting in 6-week-old infants, either in discrete-trial or in no-delay paradigms. Since evidence from a conjugate sucking paradigm suggested that the change could be discriminated by infants of this age (Morse, 1972), it seemed likely that the decelerative orienting response could be elicited only if attention were directed to a discriminable change. However, the infant no-delay procedure provided some equivocal evidence for a response of cardiac acceleration to the stimulus change. Thus, it was possible that the absence of orienting was due to the presence of a competing startledefensive response, specific to the stimuli used. The present study was undertaken, therefore, to determine whether or not adult subjects would orient to the change when, like infants, they were uninformed. The study also manipulated attention by instructing half of the subjects to listen for a change. A question of particular interest was whether instructions would have generalized effects or effects restricted to the response to change.

\section{METHOD}

Twenty-four male undergraduates at the University of Wisconsin, Madison received class credit for participating as subjects. All reported that they were free from cardiovascular, respiratory, and auditory dysfunctions and were not under the influence of drugs or medications at the time of testing. Two additional subjects were replaced because of anomalous electrocardiograms and another for excessive coughing.during the experimental session.

An IAC 404-A shielded sound-attentuated chamber furnished with a comfortable lounge chair served as the testing room. Electrocardiograms were detected with a standard Lead II configuration of Beckman biopotential electrodes leading to a Beckman Type RM dynograph outside the subject chamber. Shaped pulses coincident with each $\mathrm{R}$ wave were recorded FM on a Hewlett-Packard 3960 instrumentation recorder, and $R-R$ intervals were measured to the nearest millisecond by an offline Linc computer. Tape recorded stimuli were presented by

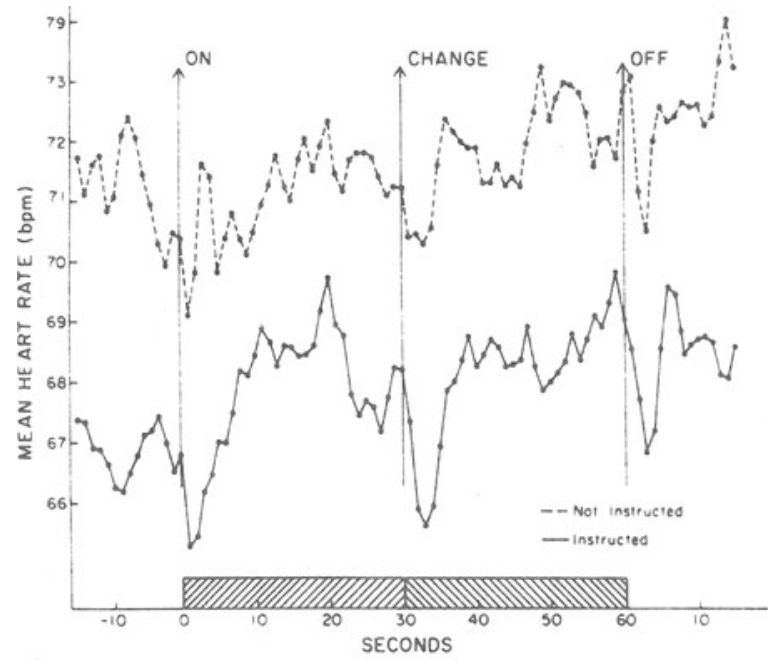

Figure 1. Mean HR from $15 \mathrm{sec}$ preceding to $15 \mathrm{sec}$ following 60 -sec trains of synthetic speech syllables changed after $30 \mathrm{sec}$. Means are based on four trials and 12 subjects per instructional group.
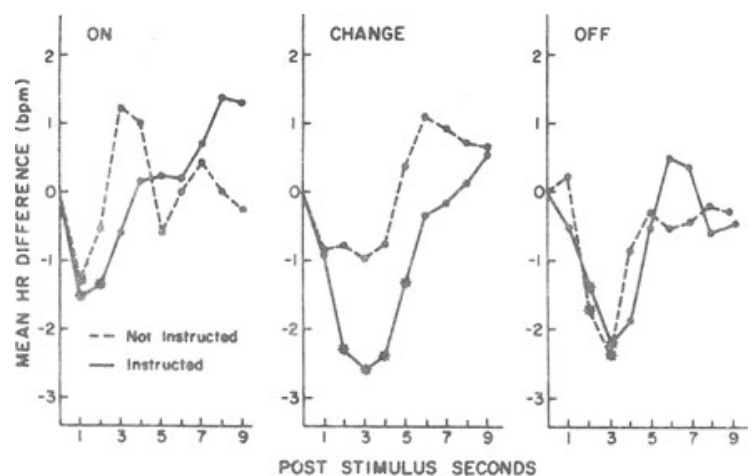

Figure 2. Mean HR response to the onset, change, and offet of 60 -sec syllable trains. Starred points indicate significant difference, at $\mathrm{p}<.05$ level, from seconds preceding stimulus manipulation.

a Sony 770 tape deck through Sharpe Mark II circumaural earphones. Intensity of stimuli delivered binaurally was $69 \mathrm{~dB}$ (A) re $20 \mathrm{~N} / \mathrm{m}^{2}$ per ear, calibrated by a Bruel and Kjaer 2203 sound-level meter with a Type $41341 / 2$-in. condenser microphone and Type 4153 artificial ear.

The stimuli and procedures replicated those employed by Leavitt et al. (Note 1) with infants. Stimuli were the threeformant syllables [ba] and [ga], generated on the Haskins Laboratories' parallel-resonance synthesizer according to the specifications described by Morse (1972). Each syllable was $500 \mathrm{msec}$ in duration and was separated from adjacent syllables in a trial by $500-\mathrm{msec}$ silent periods. A single trial consisted of 30 repetitions of one syllable followed by 30 repetitions of the other, with only the 500 -msec silent period between syllables intervening. Subjects received a total of four trials at intertrial intervals of 32 to $47 \mathrm{sec}$. Order of the initial syllable was balanced so that half of the subjects of each group received an ABBA order, i.e., [baga-gaba-gaba-baga], and half BAAB.

The first 12 subjects, the "not-instructed" group, were given instructions standard to the laboratory, i.e., they were told that they would be alone in the testing room for about $10 \mathrm{~min}$, should remain alert and move as little as possible, and would hear sounds through the earphones but were "not required to respond to them in any way." The remaining subjects, the "instructed" group, received the same instructions, with the addition of a sentence stating that the sounds would change and asking that they "listen for the change." Immediately following the period of stimulation, all subjects were questioned about what they had heard.

\section{RESULTS}

The principal findings are illustrated in Figures 1 and 2. Stimulus onset and offset elicited deceleration of approximately the same magnitude in instructed and not-instructed groups, but stimulus change produced a much larger deceleration in the instructed group. Starred points (Figure 2) indicate seconds on which HR differed significantly by $t$ test $(p<.05)$ from HR on the second preceding stimulus manipulation; even by this relaxed criterion, there was no evidence that the notinstructed group responded to the shift in speech sounds.

The main statistical analysis of response consisted of analyses of the variance in orthogonal components of trend over each of the three 10 -sec response periods, i.e., for periods including the $1 \mathrm{sec}$ preceding and the 
$9 \mathrm{sec}$ following a stimulus manipulation. This is a longer period than necessary or optimal for measuring the relatively brief cardiac orienting response of adults but was selected to maintain comparability with infant studies. Additional tests over the first 5 and over the second $5 \mathrm{sec}$ were made, as necessary, to determine whether significant linear or cubic 10 -sec trends were due to decelerative changes early or accelerative changes late in the response period. Factors included in the tests were order of presentation and instructional group (between-subject variables) and trial and sec (withinsubject variables). Trend components (linear, quadratic, and cubic seconds and linear trials) were tested by unpooled error terms, with df equal to the number of subjects less the number of groups, and were not, therefore, subject to the biasing effects of unequal covariance that are commonly found with repeated measures (Graham, 1971). Tests over sec or trials may reflect such a bias and, to compensate for this, the dfs for the denominator were also reduced in such tests to the number of subjects less the number of groups (Geisser \& Greenhouse, 1958). The only order effects interpreted were the order by quadratic trials effects, which reflected the contribution of differences between the two syllables.

Cardiac changes following stimulus onset and offset were significant and did not differ as a function of instructional groups. Onset elicited an immediate, brief deceleration followed by acceleration that produced an overall sec $F(9,20)=3.74, p<.01$, and a linear sec $\mathrm{F}(1,20)=6.80, \mathrm{p}<.05$. Additional analyses, performed over the first and over the second $5 \mathrm{sec}$ after onset, indicated that $\mathrm{HR}$ changes were reliable only during the initial $5 \sec [\sec F(4,20)=5.65, p<.01$; quadratic sec $\mathrm{F}(1,20)=15.83, \mathrm{p}<.001$; and cubic sec $\mathrm{F}(1,20)=$ $10.11, \mathrm{p}<.01]$. There was some habituation of response as a function of repeated trials [linear trials by quadratic $\sec F(1,20)=4.60, p<.05$ for the 10 -sec period and linear trials by linear sec $F(1,20)=6.69, p<.05$ for the first 5-sec period], but neither the response nor its habituation was affected by instructions or syllables. Following offset, both groups exhibited sizeable decelerations that resulted, over the $10-\mathrm{sec}$ period, in a significant sec $F(9,20)=3.28, p<.05$, and cubic sec $F(1,20)=11.16, p<.01$, and, over the first 5 -sec period, in sec $F(4,20)=6.70, p<.01$, with linear sec $\mathrm{F}(1,20)=6.51, \mathrm{p}<.05$, and quadratic sec $\mathrm{F}(1,20)=$ $5.75, \mathrm{p}<.05$. These responses did not habituate or interact with syllables or instructions.

As Figures 1 and 2 suggest, the response to stimulus change was also significant but did differ as a function of instructions. In addition to significant main effects of $\sec$ and sec trends $[\sec F(9,20)=8.49, \mathrm{p}<.001$; trend $\mathrm{Fs}(1,20)=7.66, \mathrm{p}<.05$ for linear; 22.86, $\mathrm{p}<.001$ for quadratic; and 15.94, $\mathrm{p}<.001$ for cubic], there was a significant interaction of instructions with the quadratic sec response, $F(1,20)=11.75, p<.01$. Response was not affected by syllables and did not show significant habituation.

Since HR following stimulus change provided the data of critical interest in this experiment, further analyses were carried out to determine whether a significant response occurred in each group, apart from the demonstrated difference between groups. The instructed group showed an overall sec effect $[\mathrm{F}(9,10)=12.06$, $\mathrm{p}<.001]$ as well as highly reliable components of trend [linear $\sec F(1,10)=12.49, p<.01 ;$ quadratic sec $F(1,10)=51.18, p<.001 ;$ cubic sec $F(1,10)=13.63$, $\mathrm{p}<.01$ ] . Further, applying Dunnett's multiple comparison procedure to per second changes on individual trials, significant decelerations were seen on all but the third trial. In contrast, the not-instructed group showed only a cubic trend $F(1,10)=5.21, p<.05$, over the 10 -sec period. There were no significant changes over either the first or second 5-sec period and no seconds of significant deceleration on individual trials.

The assumption underlying use of a no-delay paradigm is that HR will have recovered to a steady state in the period preceding stimulus change (Figure 1). This assumption was tested by analyzing variance in the 10-sec period preceding stimulus change in the same manner used to analyze response periods. The analysis provided no evidence of HR change during this time. Main effects of sec and sec trends were insignificant, as were their interactions with instructions, syllables, and trials. Similar analyses indicated that HR activity was also steady prior to stimulus onset and offset.

The above analyses of periods preceding and following stimulus manipulations were concerned only with phasic changes in HR, i.e., with effects of sec and interactions of sec, since it is the phasic, decelerative change that has been associated with orienting (Graham \& Clifton, 1966). Some interest also attaches to the general level of "tonic" HR. If instructions induced a generalized attentional state rather than or in addition to a specific one, HR might be expected to remain at a lower mean level in the instructed group throughout stimulation and not just during the period immediately following stimulus manipulations. This did not prove to be the case. Although mean HR was lower in the instructed than in the not-instructed group (Figure 1), averaging 68.4 as compared with 71.9 during 10 -sec periods preceding stimulus change and offset, differences did not approach significance $(\mathrm{Fs}<1.0)$ and did not change over trials.

Postexperiment questioning established that all subjects heard a change in the stimuli during each $60-\mathrm{sec}$ string. They did not all identify the stimuli as a [ba-ga] or [ga-ba] shift, but there was no clear difference as a function of instructions. The identification question was not included in questioning of the first four subjects of the not-instructed group, but 3 of the remaining 8 subjects and 6 of the 12 instructed subjects successfully named both the [ba] 
and [ga] stimuli. Furthermore, 6 of the 8 not-instructed and 7 of the 12 instructed subjects identified a difference in place of articulation along the [ba-da-ga] continuum. The remaining subjects provided a variety of descriptions, not easily summarized but including other speech syllables or one of the syllables plus some nonspeech addition such as "ping" or "thump."

\section{DISCUSSION}

The present study provides evidence that a discriminated change in stimulation does not necessarily elicit an orienting response. The subjects given no information about what they would hear and no task to perform responded minimally if at all to a stimulus change which all reported detecting. Since the same stimulus change did elicit large decelerations in instructed subjects, the particular stimulus parameters are apparently appropriate for eliciting orienting and the hypothesis suggested by our infant research, that these stimuli might be startling, appears untenable. It seems clear that, in using cardiac orienting paradigms to study discrimination, a failure to obtain orienting to a stimulus change cannot be accepted as evidence that the change is not discriminated.

It is also of interest that instructions had a substantial effect on the occurrence of orienting. The purpose of the instructions was to alter the significance to the subjects of a stimulus change without, at the same time, requiring any overt response. To the extent that the onset and offset responses of the two groups were similar, the effect of instructions was relatively precise, modifying the response to stimulus change without increasing responsivity to all stimulus events and without producing a generalized decrease in the level of HR. Further, instructions affected response to the stimulus and did not produce orienting in anticipation of it; an anticipatory effect could presumably be achieved with other types of instructions.

These observations suggest the need for caution in comparing orienting studies, since it is possible that even small differences in the subject's knowledge of the stimulus conditions or conception of the experimental task may significantly alter the results. This may be especially important when comparing studies using subjects at different developmental levels. Developmentalists have been attracted to psychophysiological measures because the similarity of responses across ages allows the use of similar experimental paradigms. The present evidence indicates that even identical paradigms may produce results that differ due to developmentally related variation in the information extracted or assumed from the total experimental situation.

\section{REFERENCE NOTE}

1. Leavitt. L. A., Brown, J. W., Morse, P. A., \& Graham, F. K. Cardiac orienting and auditory discrimination in 6-week-old infants. Manuscript in preparation, 1975.

\section{REFERENCES}

Adrinson. D. C..\& Berg. W. K. Habituation and dishabituation of cardiac orienting in newborns. Psychophysiology. 1974. 11. 219.

BERg. W. K. Habituation and dishabituation of cardiac responses in 4-month-old alert infants. Journal of Experimental Child Psychology. 1972, 14, 92-107.

Bernstein. A. S. To what does the orienting response respond? Psychophysiology. 1969, 6. 338-350.

Bernstein, A. S., Taylor, K. W., \& Weinstein, E. The phasic electrodermal response as a differentiated complex reflecting stimulus significance. Psychophysiology, 1975, 12. 158-169.

Geisser, S., \& Greenhouse, S. W. An extension of Box's results on the use of the $\mathrm{F}$ distribution in multivariate analysis. Annals of Mathematical Statistics, 1958, 29, 885-891.

Graham, F. K. Analysis of heart rate response curves: A comment on pooled interaction error terms. Psychophysiology, 1971. 7. 485-489.

Graham. F. K., \& Clifton, R. K. Heart rate change as a component of the orienting response. Psychological Bulletin. 1966. 65. 305-320.

Kagan, J., \& Lewis, M. Studies of attention in the human infant. Merrill-Palmer Quarterly, 1965, 11, 95-127.

Leavitr, L. A., Morse, P. A., Brown, J. W., \& Graham, F. K. Cardiac orienting of 6-week-old infants to speech and nonspeech stimuli. Pediatric Research, 1973, 7. 419.

Maltzman. I., \& Raskin. D. Effects of individual differences in the orienting reflex on conditioning and complex processes. Journal of Experimental Research in Personality, 1965. 1. 1-16.

Moffirt, A. R. Consonant cue perception by 20- to 24-week-old infants. Child Development, 1971, 42, 717-731.

Morse. P. A. The discrimination of speech and nonspeech stimuli in early infancy. Journal of Experimental Child Psychology. 1972, 14, 477-492.

Sokolov. E. N. Perception and the conditioned reflex. New York: Macmillan, 1963.

Sokolov, E. N. The modeling properties of the nervous system. In M. Cole and I. Maltzman (Eds.), A handbook of contemporary Soviet psychology. New York: Basic Books, 1969.

Vinogradova, O. S. On the dynamics of the orienting reflex in the course of closure of a conditioned connection. In L. G. Voronin. A. N. Leontiev, A. R. Luria, E. N. Sokolov, and O. S. Vinogradova (Eds.), Orienting reflex and exploratory behavior. Washington, D. C: American Institute of Biological Sciences, 1954. Pp. 45-53. 